
Revue
de /histoire des religions
Revue de l'histoire des religions
$3 \mid 2009$
La culture gallicane. Références et modèles (droit, ecclésiologie, histoire)

\title{
Tours contre Rome au début du règne d'Henri IV
}

Tours vs. Rome at the Start of the Reign of Henry IV

Marco Penzi

\section{(2) OpenEdition}

Journals

Édition électronique

URL : http://journals.openedition.org/rhr/7263

DOI : $10.4000 /$ rhr.7263

ISSN : 2105-2573

Éditeur

Armand Colin

Édition imprimée

Date de publication : 1 juillet 2009

Pagination : 329-347

ISBN : 978-2200-92591-8

ISSN : 0035-1423

Référence électronique

Marco Penzi, «Tours contre Rome au début du règne d'Henri IV », Revue de l'histoire des religions [En ligne], 3 | 2009, mis en ligne le 01 juillet 2012, consulté le 19 avril 2019. URL : http://

journals.openedition.org/rhr/7263; DOI : 10.4000/rhr.7263 


\section{MARCO PENZI}

Centre de recherches histEriques

(ÉcEle des Hautes Études en Sciences SEciales -

Centre NatiEnal de la Recherche Scientifique), Paris

\section{Tours contre Rome au début du règne d'Henri IV}

Pendant l'hiver 1589-90, à l'arrivée de la Légation Caetani en France et ensuite à Paris, se déchaîna une guerre d'arrêts entre les parlements ligueurs et royaux. Plus qu'une simple polémique locale entre Catholiques Unis et Politiques, cette guerre d'arrêts marqua le début d'une politique et d'un projet de séparation de l'Église de France de l'Église catholique et romaine.

\section{Tours vs. Rome at the Start of the Reign of Henry IV}

During the winter of 1589-90, at the time of the arrival of the Caetani Legation in France and subsequently in Paris, a battle of legal rulings started between the Parliament of the Catholic League and that of the Politiques. This was not just a moment of controversy between Leaguers and Royals; it also marked the beginning of a political policy and a project of separating the Church of France from the Roman Catholic one. 
Dans le journal de Pierre de l'Estoile figure un passage datant du mois de janvier 1590 :

« En ce temps on vit paraître un arrêt du Parlement séant à Tours, contre le légat Cajetan, portant défense à toutes sortes de personnes sous peine d'encourir le crime de lèse-majesté, sans espoir d'obtenir jamais la grâce, d'avoir aucune correspondance ni communication avec les ennemis de l'Etat, nommément avec le Cardinal Cajetan, jusques à ce qu'il se fût présenté au roi et au Parlement, selon les lois de la France, les droits du Royaume et les libertés de l'Eglise Gallicane $»^{1}$.

À la suite de cette information, le bourgeois parisien remarque aussi que le «parlement de Paris publia un autre arrêt quelques temps après qui cassait absolument celui donné à Tours $»^{2}$. Par ces quelques mots, L'Estoile nous fait part du conflit juridique très important qui vit, au début de l'année 1590, la Cour de parlement de Tours aux prises avec le légat pontifical, Enrico Caetani. Il s'agit aussi d'un épisode marquant de la lutte de légitimation entre les parlements royalistes et les parlements ligueurs pendant les années 1589-1594. Trois points retiennent l'attention : le moment juridique qui caractérisa le début de l'année 1590, les défenses des libertés gallicanes par le parlement de Tours, mais aussi les débuts d'un courant de pensée typique des parlementaires royalistes, qui à mon sens dépassait largement le discours habituel des légistes gallicans dans les affaires concernant les relations avec Rome, au point que même la définition de l'anti-romanisme peut sembler insuffisante ${ }^{3}$.

1. Pierre de L'Estoile, Mémoires-journaux, Paris, Tallandier, 1982, t. V, p. 265, supplément de 1736 .

2. Ibid.

3. Sur l'histoire de la légation en France de Caetani, cf. Michel de Boüard, «Sixte Quint, Henri IV et la Ligue : la légation du cardinal Caetani en France 1589-1590», Revue des questions historiques, t. 60, 1932, p. 59-140; Henri de L’Épinois, "La légation du Cardinal Caetani en France », Revue des questions historiques, année 15, t. 30, juillet 1881, p. 460-525; Henri de L'Épinois, La Ligue et les Papes, Paris, Société Générale de librairie catholique, 1886; Camillo Manfroni, «La legazione del Cardinale Caetani in Francia (1589-90) », Rivista Storica Italiana, vol. X, 1893, p. 193-270; Anne-Cécile Tizon-Germe, « Nonces et légats en France (1589-1594) », Henri IV. Le roi et la reconstruction du Royaume, Pau, 1990, p. 41-58; Anne-Cécile Tizon-Germe, «La Représentation pontificale en France au début du règne d'Henri IV (1589-1594) : cadre politique, moyens humains et financiers ", Bibliothèque de l'École de Chartes, 151, 1993, p. 37-85; 


\section{Circonstances et histoire}

Dans son Histoire, Jacques-Auguste de Thou donne une relation précise de la séance du Parlement de Paris du 26 janvier 1590, lorsque les bulles et pouvoirs du légat Enrico Caetani furent enregistrés et successivement publiés par ledit Parlement. Pendant la séance, Caetani

«... eut l'insolence de vouloir se placer sous le dais qui est reservé pour le roi; \& il auroit peut-être executé cet orgueilleux dessin, si le president Brisson, qui faisoit alors les fonctions de premier president, ne l'eût arrêté par le bras, \& ne l'eût fait descendre pour s'asseoir sur un banc au rang des autres immediatement apres lui... $»^{4}$.

De toute évidence, De Thou analyse cet événement non pas comme un geste qui est le fruit de l'ignorance de la procédure de la part d'un homme politique étranger (et donc éventuellement innocent), mais comme la tentative concrète de créer un précédent, de montrer qu'en l'absence du roi et de son représentant le plus proche, la place d'honneur mais aussi toute révérence et autorité revenaient au Pape, et le cas échéant à son représentant. Dans la reconstruction de l'historien, une fois l'accident évité, la " politique » de Caetani se dessine avec " un discours fort grave sur la puissance du Pape, \& sur les bonnes dispositions où estoit S.S. à l'égard du Royaume $\&$ du Parlement $\rangle^{5}$. Ce discours qui va de soi est la «preuve » dialectique que l'historien utilise pour démonter la volonté du geste de Caetani.

Ce fut encore Brisson, véritable alter ego fictif de tout bon gallican (et de De Thou lui-même), dans la reconstruction opérée par

Anne-Cécile Tizon-Germe, « Juridiction spirituelle et action pastorale des légats et nonces en France pendant la Ligue (1589-1594) », Archivum Historiae Pontificiae, 30, 1992, p. 159-230.

Le légat était un « ambassadeur extraordinaire de la cour de Rome. Il n'était reçu en France qu'après que le roi avait été instruit des motifs de son envoi et avait agréé sa personne. Il ne pouvait prendre les marques de sa dignité et exercer ses pouvoirs qu'après enregistrement en Parlement de ses bulles de légation et des lettres patentes du roi dont elles devaient être revêtues. Le Parlement pouvait modifier ces pouvoirs s'il croyait y voir quelque chose susceptible de porter atteinte aux Libertés de l'Eglise Gallicane, droits de la couronne, des évêques, des collateurs ». Marcel Marion, Dictionnaire des Institutions de la France, XVII ${ }^{e}$-XVIII $I^{e}$ siècles, Paris, Picard, 1999, p. 326-327.

4. Jacques-Auguste de Thou, Histoire universelle depuis 1543 jusqu'à 1607, Londres, 1734, t. XI, p. 104.

5. Ibid. 
l'historien, qui prit à nouveau la parole, pour faire un beau discours en latin " où il s'étendit fort au long sur la puissance de la France, $\&$ sur l'autorité de cette illustre compagnie $»^{6}$. La contre-harangue de Brisson sert chez de Thou, farouche ennemi de la Ligue, à introduire l'action menée en réponse par les partisans d'Henri IV. Mais on peut aussi lire dans cette reconstitution historique, qui en réalité inverse la chronologie : «Le roi opposa à ces arrêts du Parlement de Paris une déclaration qu'il adressa à la cour de parlement séant à Tours en date du 5 janvier, au sujet de l'arrivée du Cardinal Caëtano, soi-disant legat du pape ». Pour De Thou, le Parlement interpellé par le roi, selon la loi, apporta une réponse contre l'acte illégal parisien :

« il est, dit-il, parent de Boniface VIII \& frere du duc de Sermonete qui sert actuellement en Flandre dans l'armée du roi d'Espagne; ayant été envoyé par le pape Légat en France de concert avec Henri de Gusman comte d'Olivarez, il n'a choisi pour son séjour que des villes attachées au parti de la Ligue; il est entré dans le Royaume sans avoir préalablement demandé l'agrément du Roi, suivant l'usage ordinaire; \& il n'a point fait apparoître ses pouvoirs. Sur ce requisitoire, la Cour donna un arrêt par lequel elle demandoit au peuple, à la Noblesse \& au clergé d'avoir aucun commerce avec les ennemis du roi, surtout avec ledit Cardinal, \& d'entretenir avec lui aucune correspondance, soit par soi-même ou par tiers, sans la permission expresse du roi; jusqu'à ce que suivant les loix de l'estat, les droits du royaume, \& les libertés de l'eglise gallicane, ledit cardinal se soit presenté au roi conformement à l'usage establi; à peine contre les contrevenants d'être traités comme criminels de leze majesté, sans esperance de pouvoir jamais obtenir le pardon de ce crime. Donnoit au procureur general d'informer en vertu de cet arrêt contre ceux qui se montreroient refractaires aux ordres de la cour; \& de proceder même à cette recherche par la voye des censures ecclésiastiques; \& enjoignoit aux archevesques \& evesques de veiller à ce que les monitoires obtenus à cet effet fussent publiés dans leurs diocèses. [Le même jour la cour à la requisition du procureur général, donna un autre arrêt contre quiconque donneroit retraite aux rebelles, ou à ceux qui à la faveur de la guerre s'empareroient des biens des sujets du roi; declarant ceux qui se trouvaient convaincus de ce crime coupables de leze majesté; \& ordonnant de raser les chasteaux des personnes qui auroient recelé ces ennemis de l'etat $\&$ de la sureté publique] $»^{7}$.

Dans le réquisitoire au parlement, le procureur général, en exposant les faits relatifs à la légation, n'hésitait pas à attaquer person-

6. Ibid.

7. Ibid., p. 104-105. 
nellement le légat ${ }^{8}$. Caetani est ici caractérisé comme parent de Boniface VIII, mais aussi comme frère de ce duc de Sermoneta qui combattait dans les armées de Flandre : la mission du légat, incitée et voulue par l'Espagne, était privée de légitimité. Faisant référence aux libertés de l'Église gallicane, le procureur rappelait qu'un légat devait demander au souverain français la permission d'entrer dans le Royaume, comme l'avait fait quelque temps auparavant le nonce Frangipani, quand son ambassade fut refusée par Henri III9. La règle étant rappelée, il était évident que Caetani, «soi-disant légat du Pape » qui n'avait pas rempli ce devoir, était coupable. En conséquence de cette présence sans permission d'un émissaire d'une puissance étrangère en France, le parlement de Tours décrétait un arrêt par lequel il interdisait au peuple, à la noblesse et au clergé tout rapport avec le légat, que ce fût personnel ou par correspondance, direct ou indirect, ainsi que tout rapport avec les ennemis du Roi en général.

On ordonnait à Caetani de se présenter en personne devant le parlement de Tours, pour justifier sa présence en France et pour ne pas encourir d'être incriminé de lèse-majesté; il était d'ailleurs clairement dit qu'une fois condamné pour un tel crime, il n'aurait jamais obtenu le pardon. Le procureur général demandait aussi d'ouvrir des procédures à l'encontre de tous ceux qui se montraient réfractaires à l'application de cette mesure. La Cour ordonnait également aux archevêques et évêques «de proceder même à cette recherche par la voye des censures ecclésiastiques; \& enjoignoit aux archevesques \& evesques de veiller à ce que les monitoires obtenus à cet effet fussent publiés dans leurs diocèses ».

Cet arrêt du parlement de Tours constitue le premier pas des Politiques vers une nouvelle forme de gestion des affaires entre l'État et l'Église. La première mesure concernait directement le légat pontifical, accusé de lèse-majesté. Étant donné qu'il était presque certain que Caetani ne se serait jamais présenté à Tours pour se justifier,

8. Louis Servin, Recueil de ce qui fut dict par M. Servin... lors de la lecture des lettres patentes $d u$ Roy du $5^{e}$ janvier 1590, contenants déclaration de S.M. à la venue d'un des cardinauls de la cour de Rome envoyé par le Pape au royaume de France..., s. 1. n. d.

9. Cette situation est décrite par A. Lynn Martin, Correspondance du Nonce en France Fabio Mirto Frangipani (1568-1572 et 1586-1587) : nonce extraordinaire en 1574, 1575-76 et 1578, Rome, École française de Rome, Università Gregoriana, 1984, p. 22-29. 
c'est comme si le diplomate était pratiquement condamné à mort ${ }^{10}$. Les parlementaires ordonnaient aussi au clergé catholique, avec le pouvoir juridique qui leur revenait par tradition, non seulement de suivre les ordres émanant du parlement, mais aussi de prendre des mesures relevant directement de leur autorité pour agir contre le légat, en rendant publiques de telles décisions. Pour la pratique juridique française, le monitoire consistait en une injonction faite par l'autorité ecclésiastique, suivant la demande d'un juge laïc, de dévoiler tout fait concernant un crime donné sous peine d'excommunication. En France «c'étaient en général les officialités qui ordonnaient les monitoires, toujours sur l'initiative d'un juge séculier $»^{11}$.

Le parlement de Tours passait alors à une action politique de niveau supérieur. Le délit de lèse-majesté de Caetani, qui pouvait conduire tous les membres de la légation et lui-même à l'échafaud, devient compréhensible du fait que le parlement de Tours (comme les autres parlements royalistes) soutient qu'il est le seul à distribuer légitimement la justice et qu'il est la seule vraie référence juridique du Royaume. Par la condamnation de Caetani, les parlementaires de Tours justifiaient en réalité leur rôle juridique en même temps qu'ils renvoyaient dans l'illégalité l'envoyé pontifical et sa mission. Mais leur requête au clergé ajoutait une autre dimension à l'affaire. Les magistrats de Tours ordonnaient au clergé catholique (non seulement aux royalistes, mais à tous) d'agir contre Caetani. Les parlementaires confirmaient donc qu'ils étaient en mesure non seulement d'ordonner aux prélats la marche à suivre dans le domaine juridico-ecclésiastique à travers la menace de l'excommunication, mais aussi de faire juger

10. La pratique juridique française distinguait entre la lèse-majesté divine (hérésie, apostasie, sacrilège, blasphème, etc.) et humaine (attentats contre la personne du monarque ou contre l'État). Parmi les crimes de lèse-majesté humaine, on distingue deux sous-groupes : ceux « au premier chef » étaient les attentats physiques contre la personne du roi ou de ses fils, les rebellions, conspirations, etc. Les crimes de lèse-majesté " au second chef» concernaient la désertion à l'ennemi, les assemblées illicites, la fabrication des fausses monnaies, l'usurpation des fonctions publiques, la falsification du scel royal, la perception d'impôts irréguliers, etc. La majeure partie des derniers crimes était punie par la peine de mort. En revanche ceux « au premier chef» prévoyaient une morte horrible après torture; « les complices, approbateurs, étaient punis de mort : les non dénonciateurs, aussi ». Marion, Dictionnaire des institutions de France, op. cit., p. 328-329.

11. Marion, op. cit., p. 387. 
un légat, un représentant officiel, un ambassadeur du Pape, par le clergé français. En réalité, c'est la politique de Sixte V qui est condamnée lourdement.

La réponse de la Ligue eut lieu le 20 février. Le Parlement de Paris décréta un arrêt contraire à celui de Tours, en le cassant et en l'annulant. L'arrêt obligeait tous les sujets du Royaume à honorer le Saint Siège et à témoigner du respect qui était dû au légat. L'arrêt rappelait que Caetani était envoyé pour sauver la religion catholique, apostolique et romaine, et pour maintenir le peuple dans la soumission légitime qu'il doit à son roi, Charles X. La reconstruction que de Thou donne des événements est en réalité à moitié véridique et à moitié partisane : on pourrait même parler d'une manipulation de l'historien.

\section{LÉGATION ET BATAILLE DES PARLEMENTS}

Observons les détails de l'affaire. Le 18 novembre 1589 le procureur général Jacques la Guesle avertit ses collègues de l'arrivée en France d'un envoyé romain, un légat : ce prélat était vraisemblablement envoyé vers la Ligue, car les royalistes étaient tenus à l'écart de l'événement ${ }^{12}$. Informé de la chose, Henri IV écrit une lettre au parlement séant à Tours dans laquelle il

«prie messieurs les Cardinaux de Vendôme et de Lenoncourt, de s'assembler avec le parlement pour aviser tous ensemble et puis après faire à la requête du dit procureur général tout ce que l'on jugera être raisonnable à l'exemple des rois ses prédécesseurs, quand les papes ont voulu entreprendre quelque chose contre leur autorité. Et outre lui donner avis bien particulier de ce qu'il doit faire $»^{13}$.

La manœuvre d'Henri, loin d'être une requête de souverain inexpérimenté dans le gouvernement ecclésiastique du royaume ${ }^{14}$, était dictée par une grande prudence. L'arrivée d'un légat était en réalité un «test» important pour la fidélité des catholiques royalistes, et en même temps un banc d'essai de l'alliance entre le roi, les parlements politiques et les catholiques royaux. En ce sens,

12. Michel de Waele, Les Relations entre le Parlement de Paris et Henri IV, Toulouse, Publisud, 2000, p. 322.

13. Archives Nationales, X1a 91230, fol. 102.

14. De Waele, op. cit., p. 322. 
l'ordre donné à ses cousins cardinaux et catholiques est aussi une tentative de laisser démêler aux catholiques de son parti cet imbroglio religieux.

Le 20 novembre, le parlement suggère à Henri d'envoyer une personnalité d'envergure vers le légat pour lui faire part des lois du royaume et des pratiques gallicanes. La cour rappelle qu'étant le représentant d'un pouvoir étranger, le légat doit se présenter au roi légitime et non aux rebelles ligueurs. Si Caetani agit différemment, précise le parlement, toutes ses actions seront illégales, frappées de nullité, d'abus et toute procédure légale pourra être utilisée à son encontre ${ }^{15}$. Fort de cet avis, et sûr de la fidélité de ses parlements, le roi de Navarre écrit ses lettres patentes adressées au parlement de Tours le 5 janvier $1590^{16}$. Portées à la cour de parlement séant a Tours, ces lettres furent - le seize janvier, après une longue et très intéressante harangue du procureur général Servin -

« lües, publiées et enregistrées, oüy et ce requerant le procureur general du roy. Ordonne la cour que copies collationnées aux originaux seront envoyées aux baillages et senechaussées de ce ressort pour estre publiées et registrées. Enjoint aux substituts du procureur general du roy de faire proceder a la publication, et aux juges a l'execution, selon leur forme et teneur, et informe des contraventions comme de crime de leze-majesté. Et a ce que le contenu desdites lettres soit plus notoire, ordonne la cour que copies d'icelles seront affichées aux carrefours des villes et aux portes des principales églises et outre que signification en sera faite a la personne du prétendu légat, si faire se peut, et a faute de ce faire, au plus prochain lieu de leur accez de celuy auquel il est, et en ce faisant, vaudra ladite signification comme si elle estoit faite a sa personne. Fait a Tours, le seize janvier $1590 .{ }^{17}$ »

Presque en même temps, le 27 janvier on publie à Tours un arrêt qui comporte comme point principal l'interdit à toute personne de communiquer avec Caetani, sous peine de crime de lèse-majesté. Ce faisant, le parlement rendait inutile la mission du légat romain qui devait fédérer les catholiques des deux camps pour parvenir soit à la libération du cardinal de Bourbon, soit à l'élection d'un autre candidat catholique, notamment dans les personnes des deux cardinaux de la famille de Bourbon, Vendôme et Lenoncourt, qui étaient dans le camp des partisans d'Henri IV ${ }^{18}$.

15. Archives Nationales, X1a 9230, fol. 103; de Waele, op. cit., p. 323.

16. Voir le texte en annexe.

17. Dupuy, op. cit., p. 108-109.

18. Archives Nationales, X1A 9230, fol. 190. 
La réponse de la Ligue à cet arrêt n'arriva que le 20 février, avec un Arrest de la cour de parlement par lequel est enjoinct a toutes personnes de recognoistre le S. Siege et legat apostolique, contre les pretendus arrests $d u$ soy disant parlement de Tours ${ }^{19}$. L'acte émanant de Tours est « déclaré nul et sans valeur »; la cour de Parlement de Paris fait aussi « inhibitions a tous prelats seigneurs etc, de preter ayde confort et assistance à l'execution » de l'arrêt royaliste.

« Ains leur enioinct et ordonne de recognoistre et reverer le sainct siege apostolique romain, et le cardinal Caietan Legat envoyé par nostre sainct pere le pape en ce royaume, tant pour maintenir l'ancienne religion catholique Apostolique et Romaine, que pour conserver en son entier la couronne au roy, auquel naturellement et legitimement appartient : et moyenner un bon et asseuré repos en ce dit royaume, par l'estirpation des heresies, et delivrance de la personne dudict seigneur roy detenu en captivité. Faict pareillement tres expresses inhibitions et defenses a toutes personnes de quelque qualité et conditions qu'ils soient, de recevoir et publier aucuns escrites tendans au mespris dudict sainct siege, et a substraction de l'obeissance à eux deüe. Le tout sur peine d'estre declarez schismatiques, fauteurs d'heretiques et d'encourir les peines de leze majesté divine et humaine. Et aura ledit procureur general du roy commission pour faire appeler en ladicte cour tous ceux qui ont requis le contenu audict pretendu arrest, et assisté à iceluy, et autres qui par leur authorité taschent d'introduire, nourrir et fortifier l'heresie et schisme en ce Royaume, et souz-mettre le peuple à la domination d'un prince heretique, contre les Lois et Ordonnances fondamentales de l'estat receuës et iurees par tous, pour respondre à telles demandes, fins et conclusions que ledict procureur general voudra prendre contre eux $»^{20}$.

La réponse de la Ligue est forcément dure et dévoile l'enjeu de la querelle. Les parlementaires Ligueurs reconnaissent dans l'acte royaliste une soustraction d'obéissance, et perçoivent l'arrêt de Tours comme un grave danger pour l'Église de France : par le moyen d'un parlement (dont la légitimité n'est pas reconnue par le Parlement de Paris), un roi hérétique (Henri de Navarre, excommunié et donc indigne du trône) veut ou semble proposer d'ôter au Souverain Pontife tout exercice de son autorité, ou même le soumet au jugement des cours laïques françaises, en condamnant son représentant en France. Pour les Ligueurs, il est évident qu'il s'agit bien d'une tentative de schisme opérée par les parlementaires royalistes en faveur d'un roi hérétique et de ses coreligionnaires. Pour cette raison le

19. Paris, Nivelle Thierry, 1590 , avec privilège.

20. Arrest de la cour de parlement..., op. cit., p. 5-6. 
Parlement de Paris, qui invoque les Lois et Ordonnances fondamentales de l'État contre la domination d'un prince hérétique, interdit de détenir, publier ou recevoir les arrêts des parlements royalistes, sous peine de crime de lèse-majesté divine et humaine. À l'arrêt éclatant de Tours, on répond de Paris par un acte dénonciateur d'une politique schismatique, mais aussi par des mesures très sévères.

\section{L'ARRÊT DU PARLEMENT DE TOURS ET L'IDÉOLOGIE GALLICANE}

L'arrêt de Tours du 16 janvier, qui contient aussi le dossier porté par les gens du roi au réquisitoire, est défini par Maugis comme « la première manifestation publique du gallicanisme anti-romain ». Selon l'historien du Parlement, nous sommes face au « résumé de toute la politique du Parlement, au cours d'un siècle $»^{21}$. Par contre, en analysant de près le texte et la documentation utilisée par son auteur Louis Servin, on peut s'apercevoir qu'il ne confirme pas toutes les affirmations de De Thou exposées précédemment. L'arrêt du parlement de Tours est loin d'être une réponse à une provocation ligueuse, comme le soutient l'historien. Cet acte semble être le fruit d'une politique sagement menée par les Politiques gallicans : une politique qui veut démontrer qu'il existe une agression de la Ligue et de ses alliés contre les institutions du royaume, mais aussi une politique qui veut justifier la réaction de ses défenseurs les plus fermes, les parlements royalistes. Dans sa Remonstrance et conclu$\operatorname{sion}^{22}$, texte préparé pour l'occasion et qui a manifestement servi de « base » pour toute décision prise par le parlement, l'avocat général Louis Servin rappelle :

«La puissance spirituelle a esté de tout temps separée de la seigneurie temporelle, \& comme nostre seigneur lesus Christ à declaré ouvertement que son regne n'est point de ce monde, tant que l'eglise chrestienne a esté en son pur estat l'une des puissances n'a rien entre-

21. Édouard Maugis, Histoire du Parlement de Paris, Genève, Mégariotisreprints 1977, t. I, p. 721.

22. Remonstrance et conclusion [de] Monsieur l'advocat general du Roy, pour Monsieur le procureur general de sa majesté au parlement séant à Tours, le seize janvier mil cinq cens nonante, lors de la lecture des lettres patentes du roy du cinquiesme desdits mois et an, touchant le pouvoir du Pape \& de ses legats envoyez de Rome en France, dans: Louis Servin, Actions notables et plaidoyéz de Messire Louis Servin Conseiller du Roy en son conseil d'Estat, et son Advocat general en sa Cour de Parlement, Paris, Estienne Richer, 1639, p. 412 et suiv. 
pris sur l'autre: ains les Loix \& ordonnances de l'empire \& des roiaumes ont esté dissemblables, voire du tout contraires à celles de la religion. ${ }^{23}$

Le début de la Remonstrance est clair. Une nette séparation entre l'État et l'Église, voulue par le Christ lui-même, a été observée par les chrétiens des origines. Cet argument est d'autant plus efficace que Servin compare le Christ à un légat de Dieu, à un ambassadeur dont la personne est sacrée. Par contre, le Christ-légat de Dieu et les ambassadeurs comportent des différences certaines : les derniers, qui sont

« des hommes, ne parlent point aux petits, mais s'adressent aux rois, $\&$ aux premiers des peuples, vers lesquels ils sont deleguez. Cestuy (le Christ) parle à tout le monde, comme aussi est le sainct et sacré légat envoyé à la nature commune, non pour la diviser, mais pour maintenir, non par confusion, mais pour un bon ordre, comme ont fait depuis à son exemple ses apostres bien enseignez... ».

Cette Église des origines, qui était pure, a duré trois cents ans. Elle était aussi grandiose en sa souffrance quand elle a été opprimée, que le Christ lui-même auquel Servin faisait référence quelques lignes auparavant : « elle estoit triomphante, victorieuse, \& remplie de vraie felicité : et quand les premiers peres, se cachoient dans les cavernes, ils forçoient es tenebres, \& descouvroient la verité, d'autant plus que leurs ennemis taschoient à l'ensevelir $\gg{ }^{24}$. Mais ce temps glorieux ou saint est désormais révolu : dans cette reconstruction de l'histoire ecclésiastique par Servin, la faute est imputée aux princes. En reconnaissant la religion comme véritable, ils l'ont enrichie par excès d'amour avec des dons, et en l'enrichissant ont ainsi corrompu l'Église des origines. Ce fut la « grande mutation... Car le mal est entr'eux pour la primauté... ». Ce que Servin définit comme une grande mutation a été causé par une nécessité réelle : donner un ordre hiérarchique, comme divers auteurs en témoignent évoquant le fait que « Nostre sainct pere evesque de l'eglise romaine, comme successeur de saint pierre » a tenu le premier rang. Toutefois, continue Servin, « le Pape n'a eu iurisdiction ny authorité, sinon au spirituel, où il a esté recogneu le premier, mais non pas au temporel $»^{25}$. Si Servin voyait auparavant dans les largesses des souverains la

23. Servin, op. cit., p. 412.

24. Ibid.

25. Ibid. 
faute de la grande mutation, par la suite il désignait en Grégoire VII le coupable historique. Ce pape, appelé Hildebrand, a gâché un système parfait. Avant lui, l'Église originaire fonctionnait à merveille et si certains papes ont voulu " entreprendre », il suffit de se rapporter " ... à ce qu'ont fait les rois de France et quelques autres... » : ils n'ont pas toléré de telles nouveautés ${ }^{26}$. Les exemples utilisés par Servin sont nombreux : Clovis, Childebert et Theudebert, en partie laïcs et en partie clercs «par leur sacre et onction (...) pourvoyent au regime \& gouvernement de l'Eglise. Temoins les conciles d'Orléans, I et II, celui d'Auvergne où n'y étaient aucun légat du pape ». Par la suite, les rois de France ont conservé leurs prérogatives et ce système fut reconnu au point que divers autres rois étrangers s'y sont conformés.

Dans le récit de Servin, le cadre idyllique est confirmé par les intentions des « Saincts papes, ils n'ont pas esté si curieux que de vouloir sonder les secrets des Princes, ou s'ingerer en leurs hauts conseils, sinon quand ils ont esté requis, $\&$ aussi peu de leur envoyer légats pour les controller, ains ont laissé aux princes l'authorité entière... suivant l'exemple des premiers apôtres Pierre et Paul qui rendoient aux roys l'honneur qui leur est dû $\|{ }^{27}$. Servin met en avant de saints Papes qui ont suivi l'exemple des apôtres. L'avocat général présente ici une situation idéale ou idéalisée dans laquelle politique et sainteté, mais aussi État et Église sont en parfait accord.

L'exemple choisi pour décrire cette harmonie des origines est celui des lettres de Charles, maire du Palais (dit «sous l'autorité du roy ») qui donne la permission à Boniface, envoyé du pape, de venir rendre la justice en France. Suivant la pratique instaurée depuis Hugues Capet, la venue en France se fit sans permettre ni aux papes ni aux légats d'entrer ou de faire quelque chose en France au préjudice de la souveraineté temporelle ${ }^{28}$. Encore une fois, cette pratique était tellement vertueuse qu'elle fut imitée par les rois d'Angleterre, les empereurs d'Allemagne, comme les ducs de Bourgogne, les comtes de Bretagne, et bien d'autres qui l'ont adoptée dans leurs royaumes.

En faisant un pas en arrière, Servin tient à remarquer que la «grande mutation » advenue à Rome n'a pas causé de troubles

26. Ibid., p. 413.

27. Ibid.

28. Ibid., p. 413-414. 
dans la gestion des rapports entre État et Église, mais que la France a toujours étroitement surveillé la conservation des anciennes lois. Les exemples utilisés par Servin sont multiples. Il analyse en particulier ceux de Philippe le Bel et Boniface VIII, de Martin V, ainsi que les menées de Pierre de Lune « soy disant» pape Benoit XIII. Même le défunt Henri III est évoqué en citant le «cardinal Morosini, légat du pape Sixte V, duquel il prit le serment qu'il ne feroit rien contre l'estat du royaume, \& droicts de l'eglise Gallicane $»^{29}$. Le légat Morosini - exemple choisi expressément dans le récit de Servin pour décrire une situation analogue et presque contemporaine à celle de Caetani - entra en France seulement après avoir reçu la permission d'Henri III, et ses pouvoirs, selon un arrêt du 10 mars 1547, furent considérés comme valables seulement à partir du jour où l'envoyé romain fut reçu par le roi de France. D'ailleurs, conclut l'avocat général, ces mêmes pouvoirs doivent être vérifiés par le Parlement. C'est aussi à lui que sont remis, à l'issue de la légation, les registres des expéditions et le sceau, en vertu d'un arrêt de 1552 concernant le cardinal Veralde ${ }^{30}$. Ainsi, semble conclure Servin, les légats ne peuvent entrer en France sans la permission et le congé des rois, comme le démontre le précédent du cardinal de Ferrare en 1561. « Le roy s'il vouloit, pourroit courir sus à ce Cardinal Caietan, qui se qualifie aujourd'huy de Légat du Pape, puis qu'il est entré dans le Royaume sans demander la permission à sa Majesté $»^{31}$.

Cette longue reconstruction historique et juridique montre que le roi Henri IV a donc bien agi en publiant ses lettres patentes contre Caetani, un légat du Pape mais implicitement un partisan de l'Espagne. Servin donne encore un nombre considérable d'exemples de sanctions opérées par les rois contre des ambassadeurs pour le moins importuns. Cette démonstration érudite sert à l'avocat général pour rappeler que, ce faisant, Henri IV « useroit de son droict, \& non pas du sien tant seulement, ainsi du droict des gens... mais il veut sçavoir l'occasion de la venue, \& voir ce qu'il en fera cognoistre par ses departemens, $\&$ apres il advisera ce

29. Ibid., p. 416.

30. La même théorie est rappelée dans un arrêt du parlement de Paris en 1594 contre les expéditions des soi-disant légats Caetani et Sega.

31. Servin, op. cit., p. 418. 
qu'on doit faire à l'example de ses pères $\|^{32}$. Servin termine cette harangue en rappelant la « bonté » du roi encore calviniste qui veut entendre avant de juger $^{33}$.

\section{Histoire et LÉGITIMITÉ}

Nous avons vu que le récit opéré par De Thou ne concerne pas, du moins pas complètement, le texte de l'arrêt de Tours, même si par quelque artifice, l'historien montre que face à une provocation, voire à un acte illégal d'un « soy-disant » légat ${ }^{34}$, la cour de parlement assemblée à Tours répond à la requête du roi légitime, en se posant en défenseur des libertés gallicanes. Mais la reconstruction de JacquesAuguste de Thou mélange en réalité des faits différents. De Thou fait référence non au travail de l'avocat royaliste du parlement de Tours accompli pour l'arrêt du 16 janvier, mais à l'œuvre latine du même Servin imprimée plus tard cette même année ${ }^{35}$. C'est dans ce texte, écrit en latin pour qu'il ait une diffusion plus ample, que l'on retrouve la référence à Boniface VIII et à son descendent le légat Caetani ${ }^{36}$.

Jouant avec l'histoire, Servin montre qu'une fois de plus, Caetani a comme son prédécesseur attenté aux libertés gallicanes.

32. Ibid.

33. Au dossier sont ajoutés : un exemplaire de la lettre de Charles maître du palais à Boniface évêque envoyé par le pape; le formulaire du serment prêté par le cardinal Morosini sous Henri III; les facultés concédées au légat (d'Adrien VI, sous François I ${ }^{\mathrm{er}}$ ) Stephan Gabriel, évêque de Bari en 1522 (avec leurs modifications) et également celles du cardinal d'Amboise en 1501; du cardinal de Boisy en 1519; de du Part en 1529; du cardinal Veral en 1551; de Sainct George en 1553; du cardinal Caraffa en 1556; du cardinal Trivulzio en 1557; du cardinal de Ferrare en 1561 .

34. Un terme avec lequel Servin (en précisant « qui se qualifie ») définit la fonction et le rôle de Caetani, ou même lui refuse expressément ce rôle. Dans son arrêt, le Parlement l'appelait de manière similaire « prétendu légat ».

35. Louis Servin, Vindiciae secundum libertatem ecclesiae gallicanae, et defensio regii status Gallo-Francorum..., Tours, Apud J. Metayeri, 1590 (réed. 1591 et 1593$)$.

36. Ibid., p. 7 : «Et quemadmodum illorum artibus in Philippum pulchrum utriusque regni principem olim insurrexit Bonifacius VIII gente Caietanus, vir legibus nocens, falsi violatarum legum, et fidei damnatus, cuius dolis et audacia pellectius Ponfifex sanctus celestinus contra veterum canonum authoritatem de summi sacerdoti dignitate cesserat; ita eius exemplum Julius II è Rueriorum familia ligue vanissimus, vir superbo animo frustra elatus, ut Regni Franci et navarrici statum mutaret ispanorum studiosum fuit, eisque adiutor et consiliorum author extitit, et in rebus ecclesiasticis agendi ruens ac peccans...» 
Dans cette reconstruction, qui démontre que d'une mauvaise lignée ne peuvent pas sortir de bons fruits, Servin semble soutenir qu'une entreprise de très longue haleine était menée contre les intérêts de la France. Dans le nom Caetani, Servin semble voir un signe du destin, une confirmation de sa théorie selon laquelle la mutation romaine est désormais irréversible. Cette camarilla romaine, fruit d'une mauvaise graine et de sa descendance, s'adapte mal à l'esprit français, autrement dit à l'esprit des ancêtres Francs, des hommes libres comme leur nom indique. C'est cette liberté politique autant que celle de l'Église gallicane que leurs descendants se doivent de conserver ${ }^{37}$.

Dans ce même texte Servin analyse d'autres arguments qui vont dans ce sens, comme des justifications juridiques qui, trouvant leurs sources dans des textes médiévaux, servent à l'avocat pour défendre les décisions prises par Henri III contre les Guise. Servin rappelle aussi les droits au trône d'Henri IV comme roi légitime de la France. Henri IV est pour lui le roi légitime, car ce n'est pas le sacre qui crée un roi en France, mais la loi salique : la monarchie française passe d'un roi à son successeur instantanément au moment de la mort du premier. Par ailleurs, jouant par exemple avec l'histoire et les définitions, Servin rappelle une théorie qui est chère à de Thou : la nullité du Concile de Trente. En vertu du fait que ce concile n'a jamais été reçu en France, personne ne peut accuser les calvinistes d'être hérétiques. Ainsi, le seul moyen pour ramener Henri IV à la religion de ses pères est la tenue d'un libre concile. Servin souhaite qu'il soit ou bien convoqué, ou tout au moins facilité par un nouveau pape, plus enclin à écouter les positions des bons François.

L'arrêt de Tours est donc la base sur laquelle Servin a pu écrire et présenter dans un livre - qui concerne aussi bien d'autres questions - les positions gallicanes concernant l'avènement d'Henri IV et les solutions à préconiser. L'arrêt de Tours serait donc une sorte de pont entre deux positions gallicanes, l'une qui renvoie à une « tradition » établie depuis le $\mathrm{XVI}^{\mathrm{e}}$ siècle, l'autre à un développement ultérieur, dépendant des problématiques du début du règne de Henri IV. Ce développement découle aussi d'une conception de

37. Ibid., p. 1 : « Si tantus a natura majorum nostrorum mentibus libertatis amor inistus sit, ut ex eo Francorum nomen quaesierint, dedecus et flagitium magnum esset eam indolem priusquam reipublicae prodesse potuerit ex animis nostris effluxisse $»$. 
l'Église gallicane pouvant être gouvernée par son chef, un moyen de résolution possible des problèmes politiques et religieux qui ont troublé la France de cette fin de siècle. En effet, on ne pouvait chercher une solution dans l'autorité pontificale, puisque du fait de sa «mutation» elle s'était éloignée de l'esprit des saints Pères des origines du christianisme que Servin louait dans son étude préparatoire pour l'arrêt de Tours. D'ailleurs, ce fut le même Servin qui lors de la saint Martin de 1589, dans son discours au parlement de Tours, affirmait la ferme intention d'Henri IV de faire assembler un concile, vrai remède des maux de la France et des divisions entre croyants $^{38}$. Servin montrait dans cette harangue une position conciliante du roi en matière de religion, mais ce n'était qu'un moyen pour décharger la faute de la situation présente sur d'autres : ces autres « loups ravissants » ont empêché toute tentative de colloque ou de réforme par crainte de perdre leur mainmise sur les biens terrestres. Certes, Servin se fait le partisan d'une position conciliante envers les deux religions présentes en France, mais en appelant au concile, il ne défend plus une position strictement gallicane. Il ne faut pas oublier qu'Henri de Navarre était excommunié par l'Église catholique, et qu'en étant extra ecclesia il ne pouvait pas commander aux prélats (même gallicans) de s'assembler pour discuter de religion. Une telle solution n'était envisageable qu'en éliminant les positions radicales, catholiques ou protestantes; mieux : en s'éloignant de Rome, en créant une Église de France détachée de la romaine. Les positions de Servin, devenues un modèle à suivre pour les parlements royalistes en janvier 1590, ne pouvaient que susciter l'accusation de schisme, de soustraction d'obéissance, de division de l'Église catholique, ou encore d'hérésie.

Dans le cas des arrêts et lettres missives au sujet de Caetani la situation était plus claire que jamais. Envoyé en France par Sixte V, le même pape qui avait excommunié Henri de Navarre, Caetani ne se rendrait pas auprès de la cour du roi ou du parlement. Cela aurait implicitement signifié la reconnaissance de la légalité de l'un et de

38. Cf. Louis Servin, Recueil des points principaux de la harangue faicte à l'ouverture du parlement, par M. L. Servain (sic) advocat du Roy contenant exhoration aux subiects à l'obeissance envers sa majesté, Tours, Pour Claude Montr'œil et Jean Richer, 1589; id., Recueil des points principaux de la harangue faicte à l'ouverture du parlement apres la saint Martin 1589, par M. L. Servain (sic), Tours, Jamet Mettayer, 1589. 
l'autre, mais en particulier d'un souverain qui avait été privé par excommunication de tout pouvoir sur le temporel. D'autant plus que cet acte de droit canonique rendait ses sujets libres du devoir d'obéissance.

Si la Cour de parlement de Tours avait voulu vraiment se montrer conciliante, elle aurait dû organiser des entrevues avec Caetani en territoire neutre, entre catholiques, et commencer des pourparlers de paix, ou évoquer le retour du roi à la religion catholique. Toute autre initiative en provenance de Tours était vouée à la faillite. Mais les tâtonnements du cardinal de Lenoncourt, juste avant l'arrivée de Caetani, comme son refus de prendre part aux démêlées entre les parlements et le légat, sont un signal clair qu'un Bourbon catholique voulait se maintenir éloigné d'une situation qui aurait pu pour lui, cardinal catholique et possible successeur au royaume, devenir explosive.

Dans ce jeu du chat et de la souris du mois de janvier 1590, le parlement de Tours, attaquant en premier, montrait sa volonté d'affirmer la justesse de ses actions face aux crimes et aux actions condamnables des Ligueurs et de leurs alliés. Dans son Histoire, de Thou est maître de la construction de cette relation des événements qui fait toujours pencher la balance vers les bonnes et justes résolutions des fidèles d'Henri IV. L'historien mélange assez intelligemment faits historiques et textes de combat gallicans. Ce mélange, qui montre une réalité double où les bons françois par définition ne peuvent pas être de «mauvais garçons », sert les fins politiques du grand historien. Encore une fois, de Thou réussit à démontrer, preuves à l'appui, que la Ligue, avec ses entreprises favorisées par les étrangers qui veulent nuire à la France, a porté atteinte aux lois $\mathrm{du}$ royaume. Atteinte à laquelle seule une réponse, bonne et juste, peut apporter un remède. Pour ce faire, de Thou n'hésite pas à mélanger dates, événements, histoires, papes, légats, espagnols et italiens, arrêts du Parlement et droit gallicans. Voilà un exemple historique qui a connu des émules. 


\begin{abstract}
Annexe
«Lettres patentes du Roy sur la venüe du Legat» (Pierre Dupuy, Preuves de libertez de l'Eglise gallicane, s. 1., 1731, vol. 2, p. 107-108)
\end{abstract}

« Henry, par la grace de Dieu, roy de France et de Navarre, a tous ceux qui ces presentes lettres verront, salut. Comme, par bruit commun venu jusques a nos oreilles, nous ayons entendu que le Pape a depeché, il y a desja quelque temps, un de ses cardinaux qui prend qualité de legat pour venir en cestuy notre royaume, sans que nous sachions autrement a quelle fin et a quelle intention, pour ne nous en avoir esté donné aucun advis de sa part, comme il a esté observé en semblables delegations a l'endroit de roys nos predecesseurs : toutesfois, ne voulant pendant ceste incertitude de la cause de sa venuë, obmetre aucune chose qui puisse dependre de nous, soit pour ne laisser nostre intention en doute touchant ceux qui viennent de ceste part, ou pour precaution des entreprises qui pourroient estre faites, par inadvertance ou autrement, au prejudice des prerogatives de nostre royaume et des libertez de l'eglise gallicanne. Nous, pour ces causes, et autres bonnes considerations, a ce nous mouvans Avons declaré et declarons par ces presentes que si ledit Cardinal a charge de venir par devers nous, il y sera receu avec le mesme honneur qui, du tems des rois nos predecesseurs, a esté rendu a ceux de pareille qualité et tiltres que les papes ont delegué vers eux. Voulons et ordonnons audit cas a nos lieutenans generaux, gouverneurs de nos provinces et a tous autres ayans commandement de par nous, tant en icelles que particulierement en nos villes, qu'en leur faisant aparoitre de nos passe-ports, signez de nous, et de l'un de nos secretaires d'Estat, et scellez de nostre scel, lesqueles nous envoyerons audit cardinal aussitôt que nous en serons requis de sa part, ils ayent a l'accompagner ou faire accompagner, et conduire de lieux a autres, avec son train, suite, et famille, et equipage, luy donnant si bonne et forte escorte qu'il se puisse rendre seurement devers nous, ou nous entendons qu'il ait libre accez et rang, sans qu'il luy puisse en cela estre donné aucun empechement, en se comportant selon les loix, constitutions, et observations concernans la dignité et prerogatives de nostre couronne : et neanmoins, d'autant qu'il a esté de tout temps observé en notre Royaume, que nulle charge et fonction n'y peut estre exercée par les ministres et deleguez des papes, sans s'estre presentez au Roy regnant : et qu'aprez luy avoir monstré leurs pouvoirs et facultez, ils ne soient verifiez en notre cour de parlement, ou il les envoye avec ses lettres patentes pour reconnoitre s'il n'y a rien desrogeant aux droicts et libertez du royaume. Nous ne voulons souffrir que, durant nostre regne, il y soit prejudicié en aucune maniere, avons de nostre pleine puissance et authorité royale, inhibé et defendu, inhibons et defendons audit 
cardinal et a tous autres qu'il apartiendra d'entreprendre aucune charge, fonction ou office en ce royaume, souz quelques tiltres et pretextes, ou pour quelque occasion que ce soit, que prealablement les susdites formes et solemnitez n'y ayent esté gardées et observées, sur peine de nullité de tout ce qui sera fait, au contraire, comme dès à present, comme pour lors, nous avons protesté et protestons comme d'attentat et de nous pourvoir contre iceluy par toutes les voyes et manieres que nous verrons este a faire par raison. Defendons expressement a tous nos sujets, tant eclesiastiques qu'autres de quelque qualité ou dignité qu'ils soient, qu'ils n'ayent a recevoir aucun mandement, ou autres actes, n'y obeir ou avoir esgard a aucune chose procedante de luy, en vertu de quelque pouvoir que ce soit, jusqu'a ce qu'il ait apparu de nostre consentement, et aprobation, ou autorisation en la maniere sousdit, sur peine aux contrevenans d'estre tenus pour criminels de leze majesté, comme nous les declarons dès à present, attendu la qualité et importance de l'affaire, voulans qu'il soit procedé a l'encontre d'eux par voyes et peines ordinaires a semblable crimes. Et afin que la seance de nostre cour de parlement, qui a esté par le passé dans nostre ville de Paris, et autres qui nous sont a present rebelles, ne puissent servir d'excuses a aucuns, en ce qui pouroit estre expedié pour le regard de ce qui dit aucunes d'icelles, combien que l'interdiction cy-devant faite par le feu roy dernier decedé nostre tres honoré seigneur et frere, d'y exercer aucune jurisdiction, et l'establissement de nosdites cours faites en autres villes ou nostre auctorité est reconnuë leur oste toute cause de recours en ces endroits : nous avons d'abondant voulu declarer, comme nous declarons par cesdites presentes, que nulle verification, et autres expeditions qui seroient faites sur lesdits pouvoirs en aucunes desdites villes a nous rebelles ni ailleurs, autrement qu'en vertu de nos lettres patentes d'attache sur iceux ne doit estre tenuë pour bonne et valable et ne pourra servir pour descharger, ou excuser du crime susdit ceux qui y seront encourus. Si donnons en mandement a noz amez et feaux les gens tenans nosdites cours de parlement, baillifs, senechaux ou leurs lieutenans, et a tous nos justiciers et officiers a qui il apartiendra, que ces presentes ils fassent lire, publier et enregistrer et le contenu d'icelle garder, observer et entretenir inviolablement, sans y contrevenir ni souffrir estre contrevenu en aucune maniere : enjoignant a nostre procureur general en chacune de nosdites cours d'envoyer incontinent la publication qui sera faite desdites presentes copies d'icelles dûment collationnées en tous les sieges subalternes qui en dependent, pour y estre fait semblable publication, ainsi qu'il est cy-dessus mandé a tous nos officiers a qui se peut toucher chacun en son endroit. Car tel est notre plaisir. En temoin de quoy, nous avons signé ces presentes de nostre main, et en icelles fait metre notre scel. Donné au camp devant Falaize, cinq janvier 1590 et de notre regne le premier. Signé Henry. » 